\title{
EFECTIVIDAD BIOLÓGICA in vitro DE ACTINOMICETOS SOBRE EL AGENTE CAUSAL DEL TIZÓN DE HALO EN FRIJOL
}

\section{in vitro BIOLOGICAL EFFECTIVENESS OF ACTINOMYCETES UPON THE CAUSAL AGENT OF HALO BLIGHT OF COMMON BEAN}

\author{
Gabriel Rincón-Enríquez¹, Luis López-Pérez² y Evangelina E. Quiñones-Aguilar ${ }^{1 \star}$
}

\begin{abstract}
${ }^{1}$ Biotecnología Vegetal, Centro de Investigación y Asistencia en Tecnología y Diseño del Estado de Jalisco (CIATEJ). Av. Normalistas No. 800, Colinas de la Normal. 44270, Guadalajara, Jalisco. México. Tel. 01 (33) 33455200. ${ }^{2}$ Instituto de Investigaciones Agropecuarias y Forestales de la Universidad Michoacana de San Nicolás de Hidalgo. Km 9.5 Carretera Morelia-Zinapécuaro, Col. El Trébol. 58880, Tarímbaro, Michoacán, México. Tel. 01 (443) 2958323/24.
\end{abstract}

*Autor para correspondencia (eqaguilar08@gmail.com)

\section{RESUMEN}

El desarrollo de inoculantes elaborados con base en microorganismos del suelo para su empleo como agentes de control biológico de fitopatógenos, es una estrategia que podría contribuir a disminuir el uso de agroquímicos, en particular en el control de enfermedades causadas por bacterias fitopatógenas como Pseudomonas syringae pv. phaseolicola (Psph). En este estudio se evaluó in vitro la efectividad inhibitoria de 80 cepas de actinomicetos aisladas de suelos agrícolas sobre el agente causal del tizón de halo en frijol (Psph) y seleccionar las de mayor actividad antibacteriana (AAB). Para estimar el potencial de los aislados como agentes de control biológico de Psph, se evaluó su $\mathrm{AAB}$ in vitro por medio de ensayos de confrontación directa contra la cepa 1448A de Psph sobre medio de cultivo PDA (pH 7). El experimento se estableció en un diseño completamente al azar, con un total de 81 tratamientos (80 actinomicetos confrontados con Psph y un testigo comprendiendo únicamente a la bacteria fitopatógena), con tres repeticiones. La variable de respuesta evaluada fue el área de inhibición del crecimiento de Pseudomonas syringae (AIPS) determinada mediante una escala ordinal de inhibición. El análisis estadístico mediante Kruskal-Wallis presentó diferencias significativas entre cepas de actinomicetos en cuanto a su actividad antibacteriana, donde cinco cepas inhibieron por completo el crecimiento bacteriano. Estos resultados muestran el potencial de unas cepas de actinomicetos como agentes de control biológico del tizón de halo del frijol provocado por la bacteria fitopatógena Psph.

Palabras clave: Phaseolus vulgaris, Pseudomonas syringae pv. phaseolicola, antagonismo in vitro.

\section{SUMMARY}

The development of inoculants based on soil microorganisms for use as biological control agents of plant pathogens is a strategy that may reduce the use of agrochemicals, particularly for controlling diseases caused by pathogenic bacteria such as Pseudomonas syringae pv. phaseolicola (Psph). This study evaluated the in vitro inhibitory effectiveness of 80 actinomycete strains isolated from agricultural soils against the causal agent of halo blight in bean (Psph) and selected the highest antibacterial activity (ABA). To estimate the effectiveness of isolates as potential agents for the biological control of Psph, in vitro antibacterial activity was evaluated by direct confrontation against Psph strain 1448A on PDA culture medium ( $\mathrm{pH} 7$ ). The experiment was established in a completely randomized design, with 81 treatments (80 actinomycetes confronted with Psph and a control comprising only the phytopathogenic bacteria), with three replications. The recorded response variable was the inhibition growth area of Pseudomonas syringae (IAPS) determined by an ordinal inhibition scale. Statistical analysis by Kruskal -Wallis reported significant differences between actinomycetes strains on ABA, and five of them inhibited absolutely bacterial growth. The results suggest the potential of actinomycetes as biocontrol agents for bean halo blight caused by Psph.

Index words: Phaseolus vulgaris, Pseudomonas syringae pv. phaseolicola, in vitro antagonism.

\section{INTRODUCCIÓN}

El frijol (Phaseolus vulgaris L.) ocupa el segundo lugar por superficie cultivada en México. La mayor importancia de este cultivo radica en el papel que desempeña en la economía de los agricultores y como fuente vital de proteínas para la mayoría de la población mexicana. A pesar de ser un producto necesario, la producción de frijol en México es sensible a las condiciones ambientales, a problemas fitosanitarios y al desarrollo y transferencia de tecnología (SDR, 2007). En el caso específico del frijol producido en condiciones de temporal (secano), una enfermedad que se presenta con frecuencia es el tizón de halo (Campos, 1987) provocada por Pseudomonas syringae pv phaseolicola (Psph). Esta enfermedad ocurre con frecuencia en regiones con clima templado y periodos de lluvias definidos y abundantes, por ejemplo en los Estados de Aguascalientes, Zacatecas, Durango, Chihuahua, Sinaloa, San Luis Potosí, Morelos, Veracruz, Querétaro, Hidalgo y el Estado de México (Navarrete y Acosta-Gallegos, 2000). En Michigan, Estados Unidos, se estimó una pérdida en rendimiento de 23 a $43 \%$ por efecto del tizón de halo (Schwartz, 1989).

Este problema del frijol en México conduce a rendimientos bajos e inestables, que podría solucionarse mediante investigación y desarrollo y transferencia de tecnologías. El desarrollo de tecnologías podría incluir el diseño de nuevas estrategias de control biológico de enfermedades que afectan a los cultivos agrícolas y perjudican directamente a los productores, para reducir las pérdidas económicas de estos 
y a la vez disminuir el impacto ambiental.

En general, el control biológico de enfermedades en plantas se refiere al uso de organismos o sus productos para el combate de problemas fitosanitarios provocados principalmente por bacterias, hongos y nematodos. Los actinomicetos, por la gran diversidad de antibióticos y otros metabolitos bioactivos que producen, representan un grupo prometedor de microorganismos para utilizarse como agentes de control de fitopatógenos. Con base en lo anterior, el objetivo de este estudio fue evaluar in vitro la efectividad inhibitoria de 80 cepas de actinomicetos aisladas de suelos agrícolas de los Estados de Michoacán y Aguascalientes sobre el agente causal del tizón de halo en frijol (Pseudomonas syringae pv. phaseolicola) y seleccionar las de mayor actividad antibacteriana.

\section{MATERIALES Y MÉTODOS}

La bacteria fitopatógena empleada en las pruebas de actividad antibacteriana fue Pseudomonas syringae pv. phaseolicola cepa 1448A (Psph) raza 6 (Mansfield et al., 1994).

\section{Aislamiento de actinomicetos del suelo}

El aislamiento de actinomicetos nativos se hizo a partir de dos suelos agrícolas del Estado de Michoacán, El Limón (EL) y Cerro del Metate (CM) de rizosfera de agave (Agave spp.); y de un suelo del Estado de Aguascalientes Cosío (CS) (Cuadro 1), por medio de la técnica de diluciones decimales seriales y siembra en placa de agar. La primera dilución de cada muestra se realizó con el equivalente a 10 g de suelo seco en $90 \mathrm{~mL}$ de agua destilada estéril (dilución $\left.10^{-1}\right)$, y posteriormente se hicieron diluciones decimales progresivas para obtener las diluciones $10^{-2}, 10^{-3}, 10^{-4}$ y $10^{-5}$. Las diluciones $10^{-2}, 10^{-3}, 10^{-4}$ y $10^{-5}(100 \mu \mathrm{L})$ se sembraron en placas de agar de 90 x $15 \mathrm{~mm}$ que contenían Papa Dextrosa Agar (PDA-DIFCO) como medio de cultivo, a un pH de 8.5. La purificación de cepas consistió en hacer al menos tres resiembras de cada actinomiceto aislado.

\section{Confrontación in vitro de actinomicetos contra Pseudomonas syringae pv. phaseolicola}

La actividad inhibitoria de los actinomicetos contra Pseudomonas syringae pv. phaseolicola se determinó in vitro por medio de un experimento de confrontación directa. El medio de cultivo empleado fue PDA pH 7, en placas Petri de $90 \times 15 \mathrm{~mm}$. La siembra de cada actinomiceto se hizo sobre el medio de cultivo por estría en el centro de la caja $(1 \mathrm{~cm})$, y la cepa bacteriana se sembró a un lado de cada actinomiceto sobre una superficie de $2 \times 2 \mathrm{~cm}$ al dispersar con espátula de Drigalsky $10 \mu \mathrm{L}$ de un cultivo bacteriano de $16 \mathrm{~h}$ de crecimiento, ajustada a una densidad óptica $(600 \mathrm{~nm})$ de 2 , equivalente a aproximadamente $2 \times 10^{8} \mathrm{UFC} \mathrm{ml}^{-1}$ de cultivo (Figura 1). Las pruebas de confrontación entre actinomicetos y Psph se realizaron con 80 aislados (ABV01-ABV80), y el testigo comprendió únicamente a la bacteria fitopatógena. Después de $5 \mathrm{~d}$ en incubación a $26^{\circ} \mathrm{C}$, la inhibición de Psph se evaluó cuando las placas correspondientes al testigo presentaban crecimiento total sobre la superficie del medio de cultivo.

\section{Determinación de la actividad antibacteriana de Psph por medio de actinomicetos}

Para determinar la actividad antibacteriana, la variable de respuesta evaluada fue el área de inhibición del crecimiento de Psph (AIPS) por efecto de los distintos actinomicetos, de acuerdo con la escala ordinal propuesta en la Figura 2.

\section{Diseño experimental y análisis estadístico}

Los 81 tratamientos se distribuyeron en un diseño completamente al azar que incluyen al tratamiento testigo que contenía únicamente a la bacteria fitopatógena, con tres repeticiones por tratamiento. Con los datos obtenidos del área de inhibición (AIPS) se realizó un análisis estadístico mediante una prueba no paramétrica de Kruskal-Wallis e intervalos de confianza a $95 \%$ de la mediana mediante el programa Statgraphics (2005).

Cuadro 1. Localización geográfica de los sitios de muestreo de suelo empleados para el aislamiento de actinomicetos.

\begin{tabular}{|c|c|c|c|c|}
\hline Clave de la muestra & Sitio de muestreo & Municipio & Ubicación geográfica & Altitud (m) \\
\hline CS & Parcela 6 & Cosío, Aguascalientes & $\begin{array}{c}22^{\circ} 21^{\prime} 43.0^{\prime \prime} \mathrm{N} \\
102^{\circ} 16^{\prime} 52.0^{\prime \prime} \mathrm{O}\end{array}$ & 2000 \\
\hline $\mathrm{CM}$ & Cerro del Metate & Tzitzio, Michoacán & $\begin{array}{c}19^{\circ} 34^{\prime} 22.3^{\prime \prime} \mathrm{N} \\
100^{\circ} 56^{\prime} 27.5^{\prime \prime} \mathrm{O}\end{array}$ & 1915 \\
\hline LM & El Limón & Morelia-Madero, Michoacán & $\begin{array}{r}19^{\circ} 32^{\prime} 21.4^{\prime \prime} \mathrm{N} \\
101^{\circ} 05^{\prime} 41.8^{\prime \prime} \mathrm{O}\end{array}$ & 1886 \\
\hline
\end{tabular}




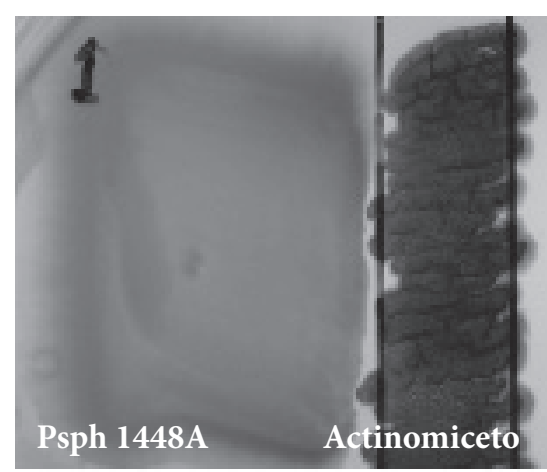

Figura 1. Crecimiento in vitro de la bacteria Pseudomonas syringae pv. phaseolicola (Psph) en el área de siembra $\left(4 \mathrm{~cm}^{2}\right)$ y del actinomiceto sembrado por estría.

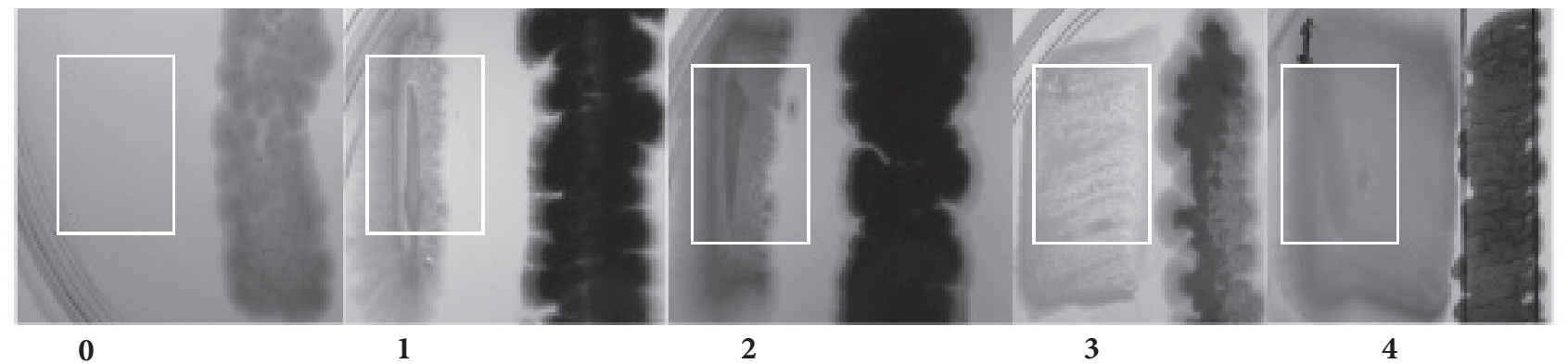

Figura 2. Escala ordinal de inhibición bacteriana por efecto de los actinomicetos. Grado: $0=(75$ a $100 \%), 1=(50$ a $75 \%), 2=$ (25 a $50 \%), 3=(0$ a $25 \%)$, y $4=0$ \% de inhibición. En los rectángulos delineados en blanco se muestra el nivel de inhibición.

\section{RESULTADOS Y DISCUSIÓN}

\section{Actinomicetos aislados de suelos agrícolas}

El crecimiento de actinomicetos se empezó a detectar una semana después de la siembra, y se observó un total de 119 cepas potenciales. Las colonias presentaron la morfología típica de este grupo microbiano: apariencia correosa y pulverulenta, con micelio muy fino aferrado firmemente al medio de cultivo (Figura 3). Luego, durante la etapa de purificación se descartaron 39 aislados por considerarse repetidos o presentar morfología de otro tipo de microorganismo, de modo que para las pruebas de actividad antibacteriana quedaron únicamente 80 aislados, de los cuales 35 provenían de El Limón, 42 de Cerro del Metate y tres de Cosío. La mayor cantidad de actinomicetos se aisló de suelo rizosférico de agave y la menor cantidad se aisló de un suelo agrícola que estuvo cultivado con chile (Capsicum annuum L.) en un ciclo anterior.

\section{Actividad antibacteriana de actinomicetos contra Psph}

El análisis de varianza no paramétrico Kruskal-Wallis mostró diferencias estadísticas significativas $(P \leq 0.01)$ entre cepas de actinomicetos para la variable AIPS. Estos resultados revelaron que los actinomicetos ABV07, ABV53, ABV55, ABV65 y ABV79 inhibieron completamente el crecimiento de Psph (valor de 0 en la escala ordinal de inhibición (Figura 4), los cuales podrían utilizarse en el desarrollo de productos para el control de la bacteria fitopatógena Psph.

De los 80 actinomicetos evaluados, 14 inhibieron en más de $50 \%$ al crecimiento de Psph, de los cuales cinco lo inhibieron en $100 \%$, cuatro en $75 \%$ y cinco en $50 \%$ (Cuadro 2). El-Abyad et al. (1992) también reportaron un efecto positivo de actinomicetos sobre el control de enfermedades causadas por las bacterias Clavibacter michiganensis y Pseudomonas solanacearum en tomate (Solanum lycopersicum L.). Sin embargo, este tema que ha sido poco explorado en México, donde la mayoría de los estudios con actinomicetos se han enfocado al control de hongos (Dávila-Medina et al., 2013). En la Figura 5 se muestra la cepa ABV65 que inhibió totalmente el crecimiento de Psph, en contraste con la cepa ABV70 que no la inhibió.

La utilización de actinomicetos como agentes de control biológico ha sido efectivo contra varios fitopatógenos, y algunos 


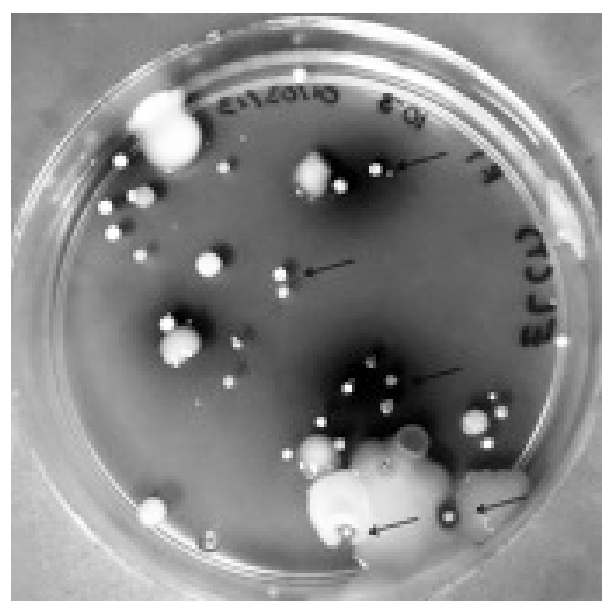

Figura 3. Colonias de actinomicetos (algunas señaladas con flechas) aisladas de El Limón Michoacán.

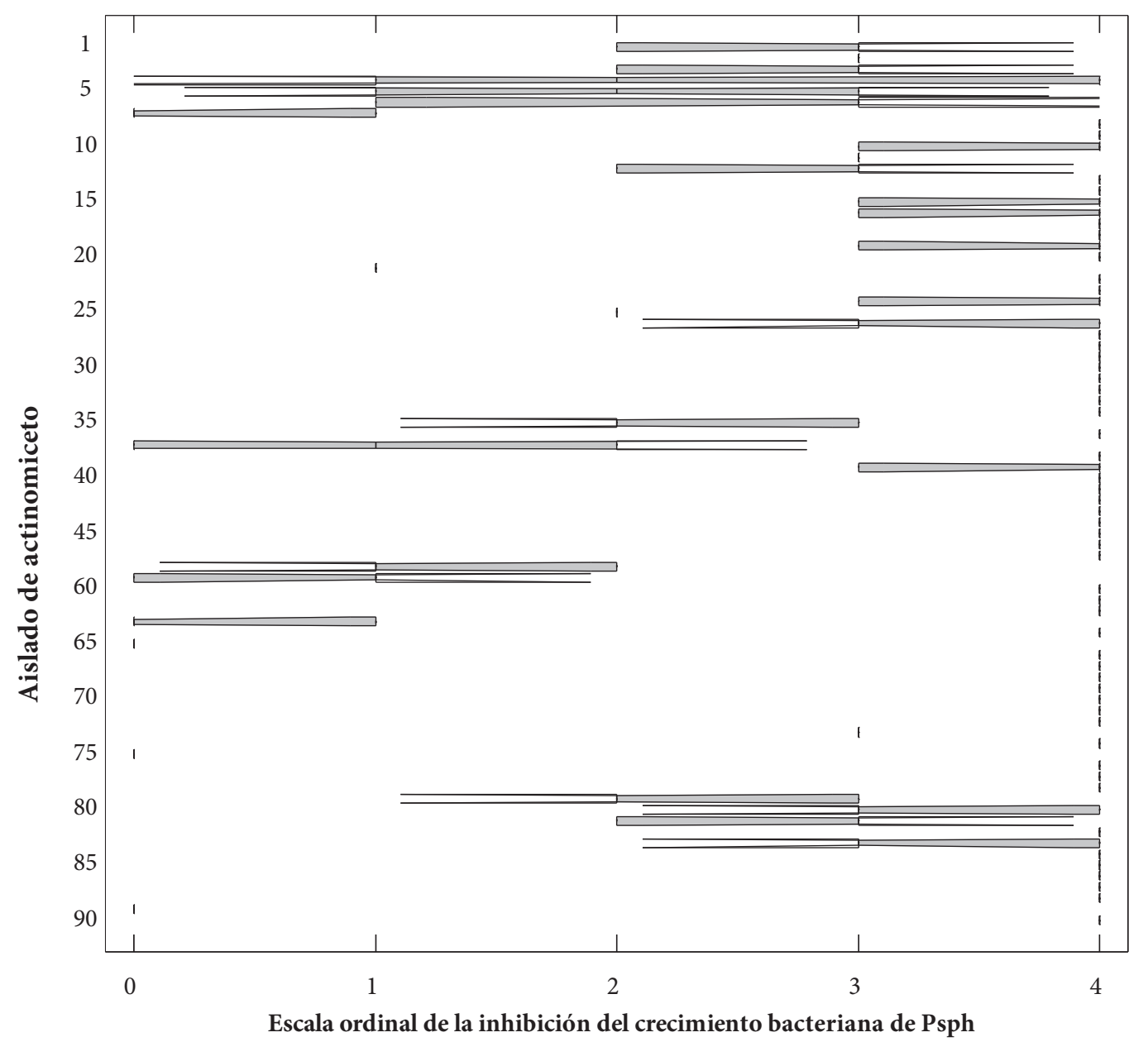

Figura 4. Efecto de 80 cepas de actinomicetos sobre el crecimiento bacteriano de Pseudomonas syringae pv. phaselicola cepa 1448A (Psph), agente causal del tizón de halo de plantas de frijol común (Phaseolus vulgaris L.). La prueba de Kruskal-Wallis produjo un estadístico de 216.3 con un valor de probabilidad de $\mathrm{P} \leq \mathbf{0 . 0 0}$. Las líneas prolongadas de las medianas indican un intervalo de confianza a un nivel de significancia de $\mathbf{P} \leq \mathbf{0 . 0 5}$. Las medianas de inhibición bacteriana se indican con líneas horizontales en cada cepa. 
Cuadro 2. Actinomicetos (ABV) con actividad antibacteriana o inhibitoria del crecimiento in vitro de Pseudomonas syringae pv. phaseolicola (Psph), mayor o igual a $50 \%$.

\begin{tabular}{lccccc}
\hline \multirow{2}{*}{ Aislado } & Origen del & \multicolumn{3}{c}{ Escala ordinal de inhibición } & \multicolumn{2}{c}{$\begin{array}{c}\text { Porcentaje de inhibición del } \\
\text { actinomiceto }\end{array}$} & Mediana & Mínimo & Máximo & 50 \\
\cline { 2 - 5 } crimiento de Psph
\end{tabular}

Sitios de muestreo: $\mathrm{CM}=$ Tzitzio; EL = Morelia-Madero, Michoacán; CS = Cosío, Aguascalientes.

productos elaborados con base en esporas de actinomicetos se comercializan como biofungicidas, principalmente del genero Streptomyces; ejemplos de estos productos son, ACTINOVATE ${ }^{A G}$ formulado con base en esporas de la cepa Streptomyces lydicus, y Mycostop ${ }^{\circledR}$ con base en Streptomyces griseoviridis, recomendados para el control de diversos fitopatógenos, principalmente hongos. Productos de este tipo son efectivos contra diversos fitopatógenos causantes de enfermedades en cultivos de importancia comercial, como chile (Capsicum spp.), fresa (Fragaria $x$ ananassa), uva (Vitis vinifera L.), gerbera (Gerbera spp.), tomate y algunas especies de la familia Cucurbitaceae, entre otros.

Diversos trabajos de investigación han mostrado que los actinomicetos son agentes promisorios para el control biológico de hongos y de bacterias fitopatógenas. Los efectos de antagonismo microbiano por parte de actinomicetos del género Streptomyces contra microorganismos fitopatógenos, se han documentado en S. rochei contra Phytophthora que afecta al cultivo del chile (Ezziyyani et al., 2007), y en $S$. griseoviridis contra Fusarium que afecta al tomate (Minuto et al., 2006).

Boukaew et al. (2011) aislaron y evaluaron la actividad inhibitoria de 265 cepas de Streptomyces spp. contra Sclerotium rolfsii causante de la pudrición de raíz y tallo en chile, y los 14 mejores aislados fueron también probados contra Ralstonia solanacearum, causante de la marchitez bacteriana en chile; los autores encontraron tres cepas efectivas contra ambos fitopatógenos, una identificada como S. $m y$ carofaciens y dos como S. philanthi. Los actinomicetos también han sido probados como promotores de crecimiento vegetal, lo que constituye un beneficio adicional en su utilización por los productores agrícolas, además de su uso como biofertilizantes que pueden ser una alternativa viable al empleo de fertilizantes químicos.

\section{CONCLUSIONES}

De las 80 cepas de actinomicetos aisladas de suelos agrícolas de Michoacán y Aguascalientes catorce (17.5\% del total) presentaron actividad antibacteriana contra Pseudomonas syringae pv. phaseolicola, con una inhibición del crecimiento in vitro de la bacteria igual o superior a $50 \%$, y de éstas cinco cepas (6 \%) causaron inhibición absoluta. Estos resultados muestran el potencial de estos actinomicetos como agentes de control biológico del tizón de halo en frijol, que se lograría por medio de una formulación diseñada especialmente para ser asperjada al follaje, o aplicada al suelo donde está el reservorio de la mayoría de bacterias fitopatógenas.

\section{AGRADECIMIENTOS}

Al financiamiento recibido mediante el Proyecto AGS2011-02-181930 del FOMIX-Gobierno del Estado de Aguascalientes-CONACYT. Al Dr. John Mansfield del "Imperial College of London, UK”, por haber proporcionado la 


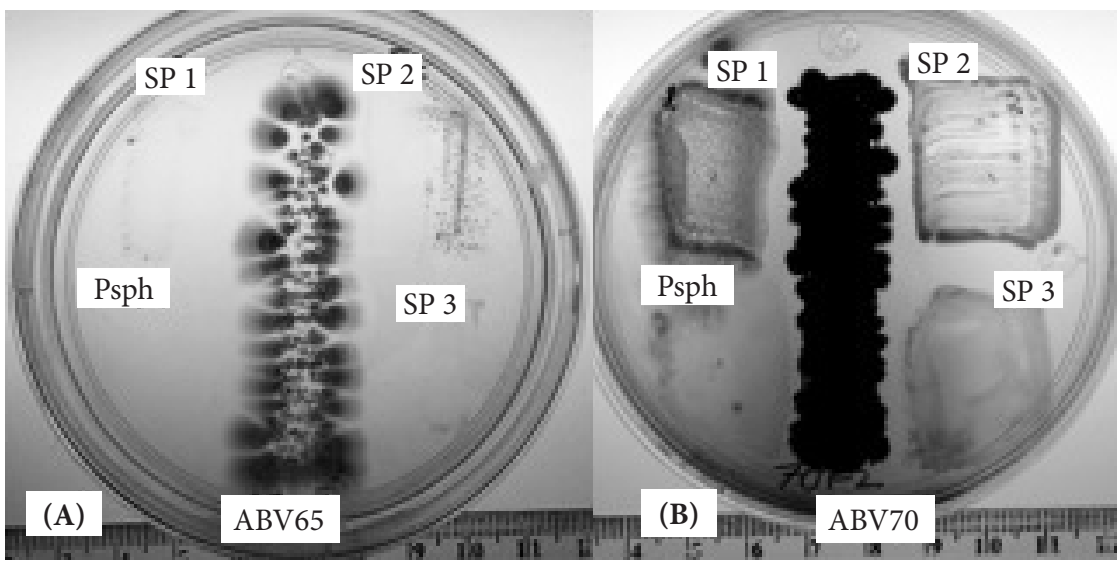

Figura 5. Actividad antibacteriana contrastante de dos actinomicetos contra Pseudomonas syringae pv. phaseolicola (Psph) en condiciones in vitro. (A) Aislado con $100 \%$ de inhibición; (B) Aislado con $0 \%$ de inhibición, en tres especies bacterianas no identificadas (Sp 1, Sp 2 y Sp 3) y en Psph.

cepa bacteriana Pseudomonas syringae pv. phaseolicola cepa 1448A (Psph) raza 6, utilizada en este estudio.

\section{BIBLIOGRAFÍA}

Boukaew S., S. Chuenchit and V. Petcharat (2011) Evaluation of Streptomyces spp. for biological control of Sclerotium root and stem rot and Ralstonia wilt of chili pepper. BioControl 56:365-374.

Campos A. J. (1987) Enfermedades de Frijol. Ed. Trillas, México. 132 p.

Dávila-Medina M. D., G. Gallegos M., F. D. Hernández C., Y. M. Ochoa F. y A. Flores O. (2013) Actinomicetos antagónicos contra hongos fitopatógenos de importancia agrícola. Revista Mexicana de Ciencias Agrícolas 4:1187-1196.

El-Abyad M. S., M. A. El-Sayerd, A. R. El-Shanshoruy and S. M. ElSabbagth (1993) Towards the biological control of fungal and bacterial diseases of tomato using antagonistic Streptomyces spp. Plant and Soil 149:185-195.

Ezziyyani M., M. E. Requena, C. Egea-Gilabert and M. E. Candela (2007) Biological control of phytophthora root rot of pepper using Trichoderma harzianum and Streptomyces rochei in com- bination. Phytopathology 155:342-349.

Mansfield J., C. Jenner, R. Hockenhull, M. A. Bennett and R. Stewart (1994) Characterization of avrPphE, a gene for cultivar-specific avirulence from Pseudomonas syringae pv. phaseolicola which is physically linked to $h r p Y$, a new hrp gene identified in the halo-bright bacterium. Molecular Plant-Microbe Interactions 7:726-739.

Minuto A., D. Spadaro, A. Garibaldi and M. L. Gullino (2006) Control of soil borne pathogens of tomato using a commercial formulation of Streptomyces griseoviridis and solarization. Crop Protection 25:468-475.

Navarrete M. R. y J. A. Acosta-Gallegos (2000) Resistencia de frijol al tizón de halo en el valle de México y progreso de la enfermedad. Revista Fitotecnia Mexicana 23:17-28.

Schwartz H. F. (1989) Halo bligth. In: Bean Production Problems in the Tropics. H. F. Schwartz and A. Pasto-Corrales (eds.). CIAT, Cali Colombia. pp:285-301.

SDR (2007) Manual de Producción del Frijol (Phaseolus vulgaris L.) del Estado de Puebla. Secretaría de Desarrollo Rural del Estado de Puebla. México. 45 p.

Statgraphics (2005) StatGraphics Centurion: ver. XV (User manual). StatPoint, Inc. USA. 380 p. 